\title{
Hypotheken des Dualismus in der Wirtschaftsethik
}

\section{Kommentar zum Hauptbeitrag von Ingo Pies}

[1] Aufgrund langjähriger Zusammenarbeit mit Ingo Pies teile ich die grundlegenden Intentionen seiner Theoriestrategie. Daher will ich in meinem Beitrag lediglich ergänzend die philosophischen Hintergründe dieser Strategie in der gebotenen Kürze skizzieren. Ethik und Wirtschaftsethik wären gut beraten, die wichtigsten Argumente dieser langen Tradition, die bei Platon und Aristoteles beginnt (vgl. Müller 2017), zu berücksichtigen.

[2] Eine spezielle Form des von Pies kritisierten Trade-off-Denkens ist der Dualismus (vgl. Homann 2015a; 2015b). Er ist durch zwei Merkmale gekennzeichnet: Handeln sieht sich zwei Forderungen, normativen Imperativen oder Werten, gegenüber, die sich (1) weder aufeinander noch auf eine gemeinsame Wurzel zurückführen lassen und von denen (2) nur eine Seite normativ ausgezeichnet und die andere damit normativ diskreditiert wird.

[3] Bei Platon ist das die Welt der Ideen einerseits, die der sittlich heruntergekommenen empirischen Polis Athen andererseits entgegengesetzt wird - diese hatte nämlich, verführt durch demagogische Rhetorik, seinen geliebten Lehrer Sokrates zum Tode verurteilt. Platon folgert daraus, dass nur Personen, die die sittliche Einsicht in die Idee des Guten und die Gerechtigkeit als diesseitiges Korrelat der Idee gewonnen haben, als Philosophenkönige diese Einsicht in der Polis verwirklichen können und sollen. Entscheidend für das Verständnis des berühmten Höhlengleichnisses ist dabei, dass die Philosophen nach ihrer Rückkehr in die Höhle, also in die empirische Polis, von den Höhlenbewohnern ausgelacht werden, ja in Lebensgefahr geraten und nur unter unsäglicher Mühe die Gerechtigkeit als das Gute gegen die hedonistisch eingestellte Menge durchsetzen können. Solchem Denken ist eine Tendenz zu autoritärer Herrschaft einprogrammiert.

[4] Das macht Aristoteles nicht mit. Gegen seinen verehrten Lehrer Platon betont er, dass bei aller sittlichen Verderbtheit der empirischen Polis dennoch in Teilbereichen ihre auf Vernunft gegründeten Institutionen grundsätzlich einen sittlichen Fortschritt gegenüber der archaischen Welt mit ihren auf Blutsbande basierenden Rechtsvorstellungen darstellen. Aufgabe der Philosophie ist es, die Vernunft nicht jenseits der Polis, also in einer idealen, der Menge immer fremd bleibenden, quasi externen Welt anzusetzen, sondern aus der empirischen Welt gewissermaßen herauszupräparieren und von dieser immanenten Basis her die unsittlichen Züge der Polis der Kritik zu unterziehen. Anders als Platon verpflichtet aber

* Prof. em. Dr. Dr. Karl Homann, Ludwig-Maximilians-Universität München, Glockenblumenstr. 36, D-80935 München, Tel.: +49-(0)89-89501850, E-Mail: karl.homann@wcge.org, Forschungsschwerpunkte: Wirtschaftsethik, praktische Philosophie. 
Aristoteles den philosophischen Lehrer nicht auch zur politischen Implementierung der Kritik.

[5] Müller zeichnet im Einzelnen nach, dass einige Schüler der platonischen Akademie zu hohen politischen Ämtern gelangt, dann regelmäßig zu Tyrannen mutiert sind, das Gemeinwesen vor die Wand gefahren haben und in Schmach und Schande gehen mussten (vgl. Müller 2017: 35ff.). Den Schlusspunkt dieser Erfahrungen setzt das Jahr 307 v. Chr. Die »Demokraten" in Athen, also die Menge, beschließen ein Gesetz, das die Gründung einer Philosophenschule ohne Zustimmung der Volksversammlung mit der Todesstrafe (!) bedrohte (vgl. ebd. 146): Sie hatten von den alles besser wissenden und mit moralischem Anspruch auftretenden Philosophenkönigen die Nase voll. - Zur Ehrenrettung von Platon und Aristoteles ist darauf hinzuweisen, dass beide, wie ebenfalls bei Müller nachzulesen ist, lernfähig geblieben sind: In ihren Spätwerken, den »Nomoi « von Platon und der »Athenaion Politeia « von Aristoteles, rücken sie von manchen autoritären bzw. antidemokratischen Elementen ihrer klassischen Werke ab.

[6] Es scheint, dass die Welt und auch die Ethik aus dieser Geschichte nur wenig gelernt haben: Immer wieder wird von Philosophen das platonische Modell propagiert, nämlich utopische Ideale oder moralische Normen, für die unbedingte normative Geltung in Anspruch genommen wird, mithilfe politischer Macht durchzusetzen, wohingegen die Stimme des Aristoteles unter intellektuellen Meinungsführern nur wenig Widerhall findet. Nur auf drei Beispiele sei hingewiesen. Gegen Robespierre, der die Tugend mit der Guillotine durchsetzen wollte, hat der »Aristoteliker « Hegel vehement Einspruch erhoben mit der berühmten Formulierung, dass dann der Tod von Menschen so bedeutungslos werde »wie das Durchhauen eines Kohlhaupts oder ein Schluck Wassers" (Hegel 1952 [1807]: 419). Dem Kommunismus mit seinem Ideal der klassenlosen Gesellschaft war der Preis von zig Millionen menschlichen Opfern nicht zu hoch. Und selbst ein so verdienstvoller und hoch dekorierter Autor wie Hans Jonas plädiert offen für »eine wohlwollende, wohlinformierte und von der richtigen Einsicht beseelte Tyrannis « (1984: 262), um die von ihm mit absolutem Vorrang versehene Moralvorstellung durchzusetzen.

[7] All diese schlimmen Irrwege lassen sich als Ausfluss eines systematischen Dualismus im Theorieaufriss verstehen. In der Wirtschaftsethik finden sich die Spuren dieses Denkens in der systematischen Entgegensetzung von Moral und Ökonomie bzw. Ethik und Ökonomik mit dem Primat der ersteren. Die kapitalistische Wirtschaft gilt gegenüber den hehren moralischen Idealen von Freiheit, Solidarität und sozialer Gerechtigkeit als das Unwahre, als sittlich verderbt und nur den egoistischen und materiellen Interessen einzelner dienend, und die Tätigkeit der Unternehmen muss daher gebändigt, gezähmt und in die Schranken verwiesen werden.

[8] Das Gegenmodell, das bei Pies die konzeptionelle Grundlage darstellt, lässt sich auf Aristoteles zurückführen. Für diesen ist das höchste Ziel des menschlichen Lebens die Eudaimonia bzw. das gute Leben; wir würden heute vom Glück bzw. einem gelingenden Leben aller sprechen. Bevorzugte Mittel dazu sind glei- 
chermaßen Ethos und Ökonomie, modern also Moral und Marktwirtschaft bzw. Kapitalismus.

[9] Ein solcher Theorieaufriss hat drei für unseren Zusammenhang wichtige Implikationen. (1) Da Moral und Ökonomie als Mittel angesetzt sind, können und müssen wir beides, also Moral und Marktwirtschaft, als gleichermaßen unverzichtbar für die Eudaimonia aller einstufen. (2) Damit können und müssen Moral und Ökonomie gegeneinander abgeglichen - und gegebenenfalls wechselseitig (!) korrigiert - werden. Oder anders mit dem ehemaligen Ratsvorsitzenden der EKD Wolfgang Huber gesagt: Bisher haben die Ethiker immer gefragt, ob die Wirtschaft "ethikverträglich" sei; wir müssen aber auch die umgekehrte Frage stellen, ob unsere Ethik »wirtschaftsverträglich" ist (Lesch/Huber 2008: 31). Schließlich haben sich die gesellschaftlichen Strukturen seit Beginn der Neuzeit tiefgreifend verändert, was für das überkommene Ethikparadigma nicht ohne Folgen bleiben kann. (3) Trotz aller empirischen Defizite und Fehler ist die Ökonomie, also die Marktwirtschaft mit ihren Systemimperativen Wettbewerb und Gewinnstreben, als Mittel zur Beförderung des Glücks aller von vornherein als grundsätzlich sittliche Institution einzustufen. Es ist das Verdienst Hegels, die Marktwirtschaft, bei ihm die »bürgerliche Gesellschaft ", als grundlegendes Element der modernen Sittlichkeit geltend gemacht zu haben, sich aber gleichzeitig dagegen zu verwahren, dass die Wirtschaft schon die ganze Sittlichkeit sei (vgl. Hegel 1986 [1821]). Eine Fundamentalkritik am Kapitalismus verbietet sich damit, weil die zu politischer Herrschaft gelangten Moralisten immer zu Misswirtschaft mit der Folge von Hunger und Armut vieler Menschen sowie zu weitreichenden Beschränkungen der Freiheit und nicht selten zu einem menschenverachtenden Despotismus geführt haben.

[10] Es lässt sich nun nicht bestreiten, dass sich das Handeln der Menschen und besonders der Führungskräfte der Wirtschaft im Alltag prima facie widersprüchlichen, nämlich moralischen und ökonomischen, Forderungen gegenübersieht. Diese unbestreitbare Problematik ist nun aber nicht dadurch auflösbar, dass man diese Alltagserfahrung in der wirtschaftsethischen Theorie noch einmal doppelt: Dann bleiben nur die Optionen, sich auf die eine oder auf die andere Seite zu schlagen und die jeweils andere zu diskreditieren - indem die Zyniker die Moral in der Wirtschaft generell als wirklichkeitsfremd ablehnen oder indem die Moralisten den wild gewordenen Kapitalismus zähmen oder ganz abschaffen wollen oder eine willkürliche »Vermittlung « beider Seiten ohne rationale Gewichtungsfunktion vorzuschlagen. Stattdessen sollte die Theorie eine rationale »Versöhnung « beider Seiten anstreben, was systematisch dadurch ermöglicht wird, dass Moral und Ökonomie, also etwa Solidarität und Wettbewerb, konzeptionell als gleichermaßen unverzichtbare Mittel im Dienst an der Eudaimonia aller stehend angesetzt werden. Das ist die normative Überbietung des Status quo, an der Pies zentral gelegen ist; diese Wirtschaftsethik erhebt den Anspruch, gegenüber dualistischen Ansätzen nicht nur die bessere Ökonomik, sondern gleichermaßen die bessere Ethik (!) zu sein.

[11] Dabei ist vor allem darauf zu achten, dass das Denken jede Vorstellung des Ausgangs von einer Tabula rasa vermeidet. Auszugehen ist stattdessen vom Status 
quo und des in ihm bereits erreichten Standes der Sittlichkeit. Eine solche Theoriestrategie ist anspruchsvoll und mag manchem Freund der »terrible simplification « als Beschmutzung seiner hehren moralischen Ideale oder Prinzipien erscheinen. Doch aus dieser Schwierigkeit der Auseinandersetzung mit den kontingenten Möglichkeiten und Unmöglichkeiten der Realität kommen wir nicht heraus: »Vielmehr zeigt sich an dieser Stelle das Dilemma aller normativen Ansprüche, die sich mit der Welt gemein machen müssen, wenn sie sie verändern wollen." (Möllers 2015: 270)

[12] Damit kommen Kontingenzen vielfältigster Art ins Spiel. Dazu hat die neuere evolutionstheoretische und empirische Moralforschung hochinteressante und auch für die philosophische Ethik bedeutsame - Überlegungen beigesteuert (vgl. [73]). Das gar nicht mehr eigens diskutierte, sondern als selbstverständlich unterstellte Ziel ist die Kooperation aller (vgl. Tomasello 2016); philosophisch heißt das: die Eudaimonia aller. Die nicht nur anfängliche, sondern permanente Bedrohung dafür ist das Trittbrettfahren, d.h. die Ausbeutbarkeit kooperativer, moralischer (Vor-)Leistungen durch - jetzt spieltheoretisch formuliert - (potentielle) Defektierer. Nie endende Aufgabe ist es daher, dieses zu unterbinden. Ein Mittel dafür ist die Entwicklung und Internalisierung bewusster Moralität als Antrieb zum Handeln, die in der weiteren Entwicklung durch kulturelle Normen »on top « (ebd. 181) unterstützt wird. Tomasello kann sich bei der Frage, ob diese "genuin moralischen " (ebd. 219), "proximalen psychologischen Mechanismen " (ebd. 223) des Handelns systematisch mit einer strategischen »ultimaten Verursachung « (ebd. 241), also mit nicht bewussten evolutionären, im weitesten Sinne ökonomischen, Vorteilen, verbunden sein muss, nicht zu einer eindeutigen Position durchringen; die Konvergenz von moralischen und strategischen Gründen bleibt zufällig: »Warum kann ich nicht beides tun? Es gibt keine bessere Verhaltensentscheidung als eine, mit der zwei Ziele zugleich erreicht werden (...) und wann immer möglich, handle ich so, dass sie alle gleichzeitig erfüllt werden.« (ebd. 244) Die so entstandene vermeintliche Eigenständigkeit eines moralischen Bewusstseins muss aber, wie ich immer betont habe und wie auch die Hirnforschung zeigt (vgl. Homann 2015c, Roth 2015 [2007]), immer durch Vorteile im weiten Sinn unterlegt sein, wenn sie in der Realität dauerhaft wirksam und stabil bleiben soll.

[13] Tomasello betrachtet die Entwicklung der Moralität bis etwa 10.000 Jahre vor unserer Gegenwart; für die Zeit danach formuliert er nur einige kursorische Überlegungen (vgl. Tomasello 2016: 240-247). Zwar sieht er die scheinbare Widersprüchlichkeit von Wettbewerb und Kooperation und die Bedeutung der Rahmenordnung für ihre Auflösung: »Nur wenn man den kulturell-institutionellen Kontext des menschlichen Verhaltens vernachlässigt, kann man glauben, dass der Karren des Wettbewerbs das kooperative Pferd zieht.« (ebd. 241; vgl. 240f.). Aber er diskutiert nicht mehr die - für eine Wirtschaftsethik der Marktwirtschaft so zentrale - Frage, wie die Probleme anzugehen sind, die sich aus der Wiedereinführung des Systemimperativs Wettbewerb in die Kooperation der modernen demokratischen Rechts- und Verfassungsstaaten ergeben, oder anders: wenn der Imperativ Trittbrettfahren als Mittel der Kooperation eingesetzt wird. Die Wirt- 
schaftsethik ist gut beraten, wenn sie den Schutz der Moral vor Ausbeutung durch Trittbrettfahrer als grundlegende Bedingung moralischen Handelns in Rechnung stellt, ohne jedoch den so produktiven Wettbewerb moralisch zu diskreditieren und politisch so weit wie möglich vermeiden oder gar abschaffen zu wollen.

\section{Literaturverzeichnis}

Hegel, G.W.F. (1952 [1807]): Phänomenologie des Geistes, hrsg. von J. Hoffmeister, 6. Aufl., Hamburg: Meiner.

Hegel, G.W.F. (1955 [1821]): Grundlinien der Philosophie des Rechts. Mit Hegels eigenhändigen Randbemerkungen in seinem Handexemplar der Rechtsphilosophie, hrsg. von J. Hoffmeister, 4. Aufl., Hamburg: Meiner.

Homann, K. (2015a): Wirtschaftsethik: Ethik, rekonstruiert mit ökonomischer Methode, in: Aaken, D. van/Schreck, P. (Hrsg.): Theorien der Wirtschafts- und Unternehmensethik, Berlin: Suhrkamp, 23-46.

Homann, K. (2015b): Theoriestrategien der Wirtschaftsethik, in: Neck, R. (Hrsg.): Wirtschaftsethische Perspektiven X, Berlin: Duncker \& Humblot, 45-66.

Homann, K. (2015c): Das Können des moralischen Sollens I und II, in: Ethica, Jg. 23/H. 3, 243-259, und H. 4, 291-314.

Jonas, H. (1984): Das Prinzip Verantwortung. Versuch einer Ethik für die technologische Zivilisation, Frankfurt a. M.: Suhrkamp.

Lesch, H./Huber, W. (2008): Sind wir allein im Universum? Der Geist, das Geld und die Welt. Gibt es Grenten der Ökonomisierung? Hrsg. Von E.ON Energie AG, Kommunikation, München.

Möllers, C. (2015): Die Möglichkeit der Normen. Über eine Praxis jenseits von Moralität und Kausalität, Berlin: Suhrkamp.

Müller, A. (2017): Platon und Aristoteles als Wegbereiter der praktischen Philosophie. Mit einem Ausblick auf die Aktualität der beiden Klassiker als Zeugen im hermeneutischen Verfahren zur Beglaubigung moderner Rechtsstaatlichkeit, Freiburg, München: Alber.

Roth, G. (2015 [2007]): Persönlichkeit, Entscheidung und Verhalten. Warum es so schwierig ist, sich und andere zu ändern, 9. Aufl., Stuttgart: Klett-Cotta.

Tomasello M. (2016): Eine Naturgeschichte der menschlichen Moral, Berlin: Suhrkamp. 